\title{
SURGERY IN DIMENSION FOUR AND NONCOMPACT 5-MANIFOLDS
}

BY

DANIEL S. SILVER

\begin{abstract}
This paper describes a precise relationship between the problems of completing surgery in dimension four and finding boundaries for noncompact 5-manifolds.
\end{abstract}

Two outstanding problems of low-dimensional topology are completing 4-dimensional surgery and finding boundaries for noncompact 5-dimensional manifolds. Although no complete theory is known for either problem, some interesting results have been found. (See $[1,4,5,10]$ and Addendum.) In this paper we describe a complete relationship between these two problems. Consequently, knowledge of 4-dimensional surgery yields information about noncompact 5-manifolds and conversely.

All manifolds and maps in this paper are assumed to be smooth. Each manifold is provided with a basepoint and, when appropriate, basepaths will be chosen to given 2-spheres without explicit mention.

I wish to thank Gerald A. Anderson and Frank Quinn for helpful discussions. Also, I wish to thank Patrick Gilmer for his advice and encouragement during this project.

1. Statement of results. Let $(f, b):(M, \partial M) \rightarrow(Y, X)$ be a degree-one normal map in the sense of Wall [11], where $M$ is a compact connected 4-manifold and $(Y, X)$ is a Poincaré pair (not necessarily simple). If $X \neq \varnothing$, then we require that $f \mid \partial M: \partial M \rightarrow X$ induces an isomorphism of homology groups with $\Lambda=\mathbf{Z}\left[\pi_{1}(Y)\right]$ coefficients. Also, we assume that $f$ is 2-connected, $K_{2}(M)$ is a free $\Lambda$-module, and the surgery obstruction $\sigma(f, b) \in L_{4}^{h}(\pi, \omega)$ is zero.

If $H \subset K_{2}(M)$ is a subkernel, we will say that $H$ is representable in $M$ if we can find disjoint framed embedded 2-spheres $S_{1}, \ldots, S_{r} \subset$ int $M$ such that the inclusion $M-\cup_{i} S_{i} \rightarrow M$ induces an isomorphism of fundamental groups, and the classes of $S_{1}, \ldots, S_{r}$ in $H_{2}(M ; \Lambda) \cong \pi_{2}(M)$ form a basis over $\Lambda$ for $H$. The subkernel $H$ is stably representable if, for some integer $k \geqslant 0, H \oplus\left\langle e_{1}, \ldots, e_{k}\right\rangle$ is representable in $M \# k\left(S^{2} \times S^{2}\right)$. Here $e_{i}$ denotes the class of $S^{2} \times 1 \subset i$ th-summand $S^{2} \times S^{2}$, and $\left\langle e_{1}, \ldots, e_{k}\right\rangle$ is the submodule generated by $e_{1}, \ldots, e_{k}$. We regard $H \oplus\left\langle e_{1}, \ldots, e_{k}\right\rangle$ as

Received by the editors August 10, 1982.

1980 Mathematics Subject Classification. Primary 57R65; Secondary 57M99.

(C)1985 American Mathematical Society $0002-9947 / 85 \$ 1.00+\$ .25$ per page 
a subkernel after performing surgery on $(f, b)$ to " kill" $k$ null-homotopic circles in int $M$.

Now suppose that $H_{1}$ and $H_{2}$ are two subkernels of $K_{2}(M)$. Then any $\Lambda$-module isomorphism $H_{1} \rightarrow H_{2}$ extends to an automorphism $\alpha$ of $K_{2}(M)$ [11, Corollary 5.3.1]. In fact, if we identify $K_{2}(M)$ with the standard kernel, then $\alpha$ represents a well-defined element $\bar{\alpha}$ of $L_{5}^{h}(\pi, \omega)$. Assume that $H_{1}$ is representable. We can perform (relative) surgery on $(f, b)$ and obtain $\left(f^{\prime}, b^{\prime}\right)$ where $f^{\prime}: P \rightarrow Y$ is a homotopy equivalence. In the next section, we will prove the following

PROPOSITION 1. The subkernel $\mathrm{H}_{2}$ is stably representable iff there exists a normal cobordism $(F, B)$ rel $\partial$ from $\left(f^{\prime}, b^{\prime}\right)$ to $\left(f^{\prime \prime}, b^{\prime \prime}\right)$, where $f^{\prime \prime}$ is a homotopy equivalence and the surgery obstruction $\sigma(F, B)$ is precisely $\bar{\alpha}$.

COROLlaRY 2. If $\bar{\alpha}=0$, then $\mathrm{H}_{2}$ is stably representable.

REMARK. If we replace $L_{n}^{h}(\pi, \omega)$ everywhere by $L_{n}^{s}(\pi, \omega)$, then after making the obvious necessary modifications the corresponding conclusion of Proposition 1 can be obtained from the results of [1, Appendix].

Now given any degree-one normal map $(f, b)$ as above with $\sigma(f, b)=0$ in $L_{4}^{h}(\pi, \omega)$, we construct a noncompact 5-manifold $W$ with a single end $\varepsilon$, tame with vanishing collar obstruction. (See [8] for terminology.) As an application of the above proposition we prove

THEOREM 3. If $\varepsilon$ has a collar in $W$, then $(f, b)$ has a solution; i.e., $(f, b)$ is normally cobordant rel d to $\left(f^{\prime}, b^{\prime}\right)$, where $f^{\prime}$ is a homotopy equivalence.

Conversely, if $(f, b)$ has a solution $\left(f^{\prime}, b^{\prime}\right)$, then an element $\bar{\alpha}$ in $L_{5}^{h}(\pi, \omega)$ is defined. The end $\varepsilon$ has a collar in $W$ iff there exists a normal cobordism $(F, B)$ rel $\partial$ from $\left(f^{\prime}, b^{\prime}\right)$ to $\left(f^{\prime \prime}, b^{\prime \prime}\right)$ such that $f^{\prime \prime}$ is a homotopy equivalence and the surgery obstruction $\sigma(F, B)$ is precisely $\bar{\alpha}$.

COROllaRy 4. If $L_{5}^{h}(\pi, \omega)=0\left(\right.$ e.g., $\left.\pi_{1}(Y)=1\right)$, then $(f, b)$ has a solution iff $\varepsilon$ has a collar in $W$.

If $f$ is already a homotopy equivalence, then we can show

Corollary 5. Let $\beta$ be any element of $L_{5}^{h}(\pi, \omega)$. Then there exists a noncompact 5-manifold $W$ as above such that the end $\varepsilon$ has a collar in $W$ iff there exists a normal cobordism $(F, B)$ rel $\partial$ from $(f, b)$ to $\left(f^{\prime}, b^{\prime}\right)$ such that $f^{\prime}$ is a homotopy equivalence and $\sigma(F, B)=\beta$.

Finally, let $W$ be any noncompact 5-manifold such that $\partial W$ (possibly empty) is diffeomorphic to the interior of a compact manifold. Suppose that $\varepsilon$ is a tame end of $W$ with vanishing collar obstruction. We produce a degree-one normal map $(f, b)$ as above such that the conclusion of Theorem 3 is valid.

2. Proof of Proposition 1. Let $H_{1}, H_{2}$ be subkernels of $K_{2}(M)$ and suppose that $H_{1}$ is representable in $M$. Let $\alpha$ be any automorphism of $K_{2}(M)$ that restricts to an isomorphism from $\mathrm{H}_{1}$ to $\mathrm{H}_{2}$. 
LEMMA 6. If $\alpha$ is an element of $R U_{r}(\Lambda)$ and $r$ is odd, then $H_{2}$ is representable in $M$. (See [11, Chapter 6] for the definition of $R U_{r}(\Lambda)$.)

Proof. Since $H_{1}$ is representable in $M$, there exist disjoint framed embedded 2-spheres $S_{1}, \ldots, S_{r} \subset$ int $M$, as in $\S 1$, whose classes $a_{1}, \ldots, a_{r}$ in $H_{2}(M ; \Lambda)$ form a basis for $H_{1}$ over $\Lambda$. Extend $a_{1}, \ldots, a_{r}$ to a basis $a_{1}, \ldots, a_{r}, a_{1}^{\prime}, \ldots, a_{r}^{\prime}$ for $K_{2}(M)$ such that $\lambda\left(a_{i}, a_{j}\right)=\lambda\left(a_{i}^{\prime}, a_{j}^{\prime}\right)=0, \lambda\left(a_{i}, a_{j}^{\prime}\right)=\delta_{i j}$ and $\mu\left(a_{i}\right)=\mu\left(a_{i}^{\prime}\right)=0,1 \leqslant i, j \leqslant r$. Using [2, Lemma 2.7 and identities at the bottom of p. 406], we can express $\alpha$ with respect to the basis

$$
\alpha=\left(\begin{array}{cc}
R & 0 \\
R & \left(R^{*}\right)^{-1}
\end{array}\right) \cdot \alpha_{1} \cdots \alpha_{l},
$$

where $R$ is contained in $\operatorname{GL}(r, \Lambda)$ and each $\alpha_{i}$ is of the form

$$
\text { (i) }\left(\begin{array}{ll}
I & P \\
0 & I
\end{array}\right) \text { or (ii) }\left(\begin{array}{ll}
0 & I \\
I & 0
\end{array}\right) \text {. }
$$

Here $P=Q-Q^{*}$ for some matrix $Q$.

If $l=0$, then $a_{1}, \ldots, a_{r}$ generate $H_{2}$ and we are done. Assume that $l>0$. If $a_{l}$ is of type (i), then replace $a_{1}^{\prime}, \ldots, a_{r}^{\prime}$ by $\alpha_{l}\left(a_{1}^{\prime}\right), \ldots, \alpha_{l}\left(a_{r}^{\prime}\right)$, respectively. This has the effect of reducing $l$ by one. If $\alpha_{l}$ is of type (ii), then by [7] we can represent the classes $a_{1}^{\prime}, \ldots, a_{r}^{\prime}$ by framed immersed 2-spheres $S_{1}^{\prime}, \ldots, S_{r}^{\prime} \subset$ int $M$ such that $S_{i} \cap S_{j}^{\prime}=\varnothing$ if $i \neq j$ and $S_{i} \cap S_{i}^{\prime}$ consists of a single point of transverse intersection, $1 \leqslant i, j \leqslant r$. By [7, Proposition 2.2], $S_{1}^{\prime}, \ldots, S_{r}^{\prime}$ are regularly homotopic to disjoint embedded 2-spheres. Moreover, the regular homotopy can be chosen so that it does not introduce new intersection points with $S_{1}, \ldots, S_{r}$. Consequently, $S_{1}, S_{1}^{\prime}, \ldots, S_{r}, S_{r}^{\prime}$ form $r$ disjoint wedges of 2-spheres. Interchange $S_{i}$ and $S_{i}^{\prime}\left(a_{i}\right.$ and $\left.a_{i}^{\prime}\right)$. Again $l$ is reduced by one. Inductively we can assume that $l=0$.

Now assume that $H_{2} \oplus\left\langle e_{1}, \ldots, e_{k}\right\rangle$ is representable in $N=M \# k\left(S^{2} \times S^{2}\right)$. By hypothesis $H_{1}$ is representable in $M$. Choose disjoint framed embedded 2-spheres $S_{1}, \ldots, S_{r+k} \subset$ int $N$, as in $\S 1$, such that classes of $S_{1}, \ldots, S_{r}$ form a basis for $H_{1}$ while $S_{i}=S^{2} \times 1 \subset i$ th-summand $S^{2} \times S^{2}$ for $i>r$. Construct a cobordism $V_{1}$ by attaching 3-handles to $N \times I$ along these 2-spheres in $N \times 1$. Notice that $V_{1}$ is a relative cobordism between $N$ and $P$. Similarly, form a cobordism $V_{2}$ by attaching 3-handles to $N \times I$ along 2-spheres in $N \times I$ whose classes form a basis for $H_{2} \oplus\left\langle e_{1}, \ldots, e_{k}\right\rangle$. Identify $V_{1}$ and $V_{2}$ along their common boundary part $N$ in order to obtain $V$. It is possible to extend $\left(f^{\prime}, b^{\prime}\right)$ over $V$ to obtain a normal cobordism $(F, B)$ rel $\partial$. One verifies as in $[11$, p. 66] that $\sigma(F, B)=\bar{\alpha}$.

Conversely, assume that the cobordism $(F, B)$ exists where $F: V \rightarrow Y \times I$. After doing surgery on circles inside int $V$, we may assume that the inclusions $P=\partial_{-} V \rightarrow$ $V, \partial_{+} V \rightarrow V$ induce isomorphisms of fundamental groups. Consequently, by the proof of [6, Theorem 8.1] we can find a handlebody-decomposition $V=P \times I \cup$ $h_{1}^{2} \cup \cdots \cup h_{m}^{2} \cup h_{1}^{3} \cup \cdots \cup h_{m}^{3}$ containing handles of index 2 and 3 only. The number of 2-handles is equal to the number of 3-handles since $P$ and $\partial_{+} V$ are homotopy equivalent. After adding cancelling pairs of 2- and 3-handles, if necessary, we can assume that $m=r+k, k \geqslant 0$, and that $m$ is odd. Then $N \approx P \# m\left(S^{2} \times S^{2}\right)$ 
and the classes of the belt 2-spheres $S_{1}, \ldots, S_{m}$ of $h_{1}^{2}, \ldots, h_{m}^{2}$, respectively, generate a subkernel of $K_{2}(N)$. Let $H_{2}^{\prime} \subset K_{2}(N)$ be the subkernel generated by

$$
\text { ว (core 3-disk of } \left.h_{i}^{3}\right), \quad 1 \leqslant i \leqslant m \text {. }
$$

Recall that $P$ was first obtained from $M$ by performing surgery on $f:(M, \partial M) \rightarrow$ $(Y, X)$. We can write $M \approx P \# r\left(S^{2} \times S^{2}\right)$ where now $S_{1}, \ldots, S_{r}$ generate the subkernel $H_{1}$. Identify $N$ with $M \# k\left(S^{2} \times S^{2}\right)$. Then any isomorphism $H_{2}^{\prime} \rightarrow H_{2} \oplus$ $\left\langle e_{1}, \ldots, e_{k}\right\rangle$ extends to an automorphism of $K_{2}(N)$ which, for sufficiently large $m$, must be an element of $R U_{m}(\Lambda)$. Since $m$ is odd and $H_{2}^{\prime}$ is representable (using the 2-spheres $\partial\left(\right.$ core $\left.h_{i}^{3}\right)$ ) the subkernel $H_{2} \oplus\left\langle e_{1}, \ldots, e_{k}\right\rangle$ is representable in $M \#$ $k\left(S^{2} \times S^{2}\right)$ by Lemma 6 .

3. Applications to noncompact 5-manifolds. Let $(f, b):(M, \partial M) \rightarrow(Y, X)$ be a degree-one normal map such that $f$ is 2-connected, $K_{2}(M)$ is a free $\Lambda$-module and $\sigma(f, b)=0$ in $L_{4}^{h}(\pi, \omega)$. We construct a noncompact 5-manifold $W$ as follows: First choose any subkernel $H \subset K_{2}(M)$ with basis $a_{1}, \ldots, a_{r}$ such that $\lambda\left(a_{i}, a_{j}\right)=0$ and $\mu\left(a_{i}\right)=0,1 \leqslant i, j \leqslant r$. Let $U_{1}=M \times I \cup h_{1}^{2} \cup \cdots \cup h_{r}^{2}$, where each 2-handle $h_{i}^{2}$ is attached along with a null-homotopic circle in int $M \times 1$ so that $\partial_{-} U_{1}=M$ while $\partial_{+} U_{1}=N$, where $N=M \# r\left(S^{2} \times S^{2}\right)$. Using [7] we can find disjoint framed embedded 2-spheres $S_{1}, \ldots, S_{r} \subset$ int $N$ representing $a_{1}, \ldots, a_{r}$, respectively, such that the inclusion $N-\bigcup_{i} S_{i} \rightarrow N$ induces an isomorphism of fundamental groups. Let $V_{1}=N \times I \cup h_{1}^{3} \cup \cdots \cup h_{r}^{3}$, where $h_{i}^{3}$ is a 3-handle attached to $N \times 1$ along $S_{i}$ with its framing. Identify $U_{1}$ and $V_{1}$ along their common boundary part $N$ in order to obtain $W_{1}$. Then $W_{1}$ is a relative cobordism from $M$ to a 4-manifold $\partial_{+} W_{1}$ which we will denote by $M_{1}$. It is possible to extend $(f, b)$ to a normal cobordism

$$
\left(F_{1}, B_{1}\right) \text { rel } \partial \text {, where } F_{1}:\left(W_{1} ; M, N, M_{1}\right) \rightarrow\left(Y \times I ; Y \times 0, Y \times \frac{1}{2}, Y \times 1\right) \text {. }
$$

Moreover $F_{1} \mid M_{1}$ is 2-connected and $K_{2}\left(M_{1}\right)$ has a subkernel with basis $b_{1}, \ldots, b_{r}$ corresponding to the belt 2-spheres of $h_{1}^{2}, \ldots, h_{r}^{2}$. Let $H_{1}$ be a dual subkernel; i.e., a subkernel of $K_{2}\left(M_{1}\right)$ with basis $b_{1}^{\prime}, \ldots, b_{r}^{\prime}$ such that $\lambda\left(b_{j}^{\prime}, b_{j}^{\prime}\right)=0, \mu\left(b_{i}^{\prime}\right)=0$ and $\lambda\left(b_{i}, b_{j}^{\prime}\right)=\delta_{i j}, 1 \leqslant i, j \leqslant r$. Using the subkernel $H_{1}$, construct a cobordism $W_{2}=$ $U_{2} \cup V_{2}$ as before. Inductively construct $W_{i}$ and define $W=\cup_{i} W_{i}$, where $W_{i}$ and

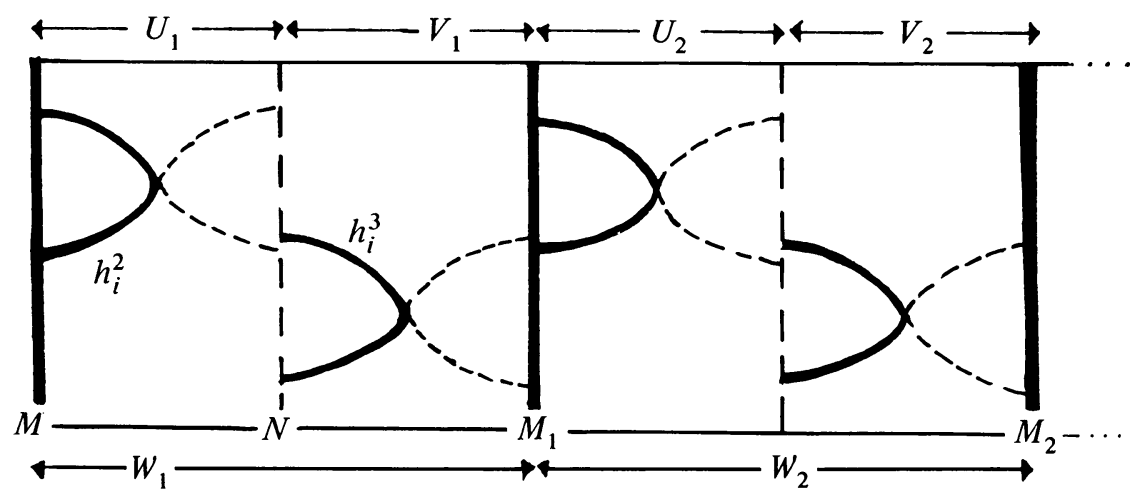

Figure 1 
$W_{i+1}$ are identified along their common boundary part $\partial_{+} W_{i} \approx \partial_{-} W_{i+1}$. (See Figure 1.) If $\partial M \neq \varnothing$, then round the resulting corners. Notice that if $\partial M \neq \varnothing$, then $\partial W \approx M \cup\{$ open collar of $\partial M\}$.

The subsets $\bigcup_{i \geqslant k} W_{i}, k \geqslant 1$, determine a unique end $\varepsilon$ of $W$. Clearly $\pi_{1}$ is stable at $\varepsilon$ and the natural maps $\pi_{1}(\varepsilon) \rightarrow \pi_{1}(W) \cong \pi_{1}(M)$ are isomorphisms. A straightforward calculation shows that $H_{q}(W, M ; \Lambda)=0$ if $q \neq 3$, while $H_{3}(W, M ; \Lambda)$ is a free $\Lambda$-module of rank $r$. In fact, the image of the boundary homomorphism $\partial$ : $H_{3}(W, M ; \Lambda) \rightarrow H_{2}(M ; \Lambda)$ is precisely the subkernel $H \subset K_{2}(M)$. It follows by [8, Lemma 6.2] that $\varepsilon$ is a tame end with vanishing collar obstruction. (This obstruction is the equivalence class of the $\Lambda$-module $H_{3}(W, M ; \Lambda)$ in $\tilde{K}_{0}\left(\pi_{1}(\varepsilon)\right)$, the projective class group of $\pi_{1}(\varepsilon)$.)

REMARK. There are other ways to construct a noncompact 5-manifold which satisfies the conclusion of Theorem 3. For example, we can cross $(f, b)$ with $S^{1}$, complete surgery and take the appropriate infinite cyclic cover. Then let $W$ be a closed neighborhood of one of the two ends.
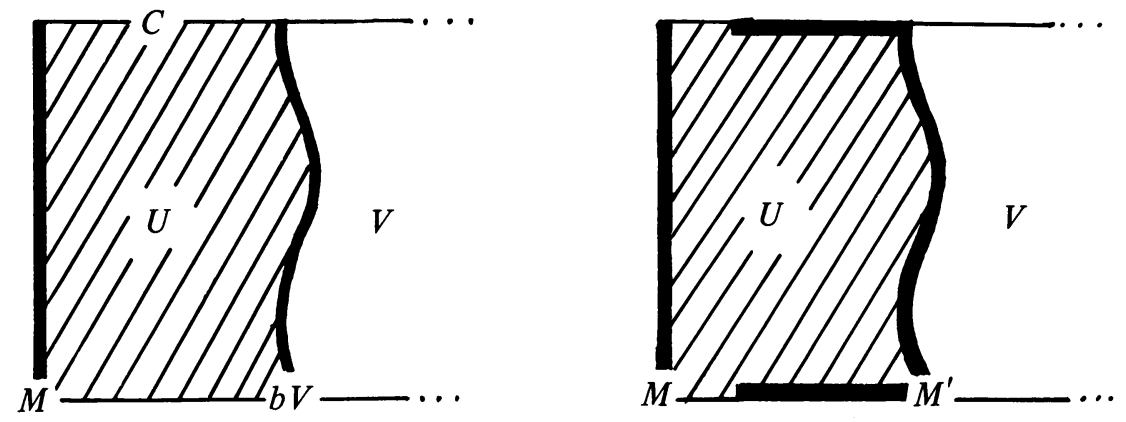

FIGURE 2

Proof of Theorem 3. Assume that $\varepsilon$ has a collar $V$ in $W$. Then $V$ is a connected neighborhood of $\varepsilon$ that is a closed submanifold of $W$. The frontier $b V$ of $V$ is a compact submanifold (possibly with boundary) and $V \approx b V \times[0, \infty)$. Let $U$ denote the closure in $W$ of $W-V$. If $\partial M=\varnothing$, then $U$ is a (relative) cobordism between $M$ and $M^{\prime}$, where $M^{\prime}=b V$. If $\partial M \neq \varnothing$, then $C=\partial U-\{$ int $M \cup$ int $b V\}$ is an $h$-cobordism between $\partial M$ and $\partial(b V)$. In this case, let $M^{\prime}=b V \cup\{C$-open collar of $\partial M\}$. Then $U$ is again a relative cobordism between $M$ and $M^{\prime}$. (See Figure 2.)

It is easy to verify that the inclusions $M \rightarrow U, M^{\prime} \rightarrow U$ induce isomorphisms of fundamental groups. Consequently, we can find a handlebody decomposition

$$
U=M \times I \cup k_{1}^{2} \cup \cdots \cup k_{s}^{2} \cup k_{1}^{3} \cup \cdots \cup k_{t}^{3}
$$

containing handles of index 2 and 3 only. Let $N$ denote the middle-level 4-manifold. Extend $(f, b)$ to a normal cobordism

$$
(F, B) \text { rel } \partial, \quad \text { where } F:\left(U ; M, N, M^{\prime}\right) \rightarrow\left(Y \times I ; Y \times 0, Y \times \frac{1}{2}, Y \times 1\right) .
$$

We claim that $(F, B)$ is a normal cobordism from $(f, b)$ to $\left(f^{\prime}, b^{\prime}\right)$ where $f^{\prime}$ : $M^{\prime} \rightarrow Y$ is a homotopy equivalence. To see this, let

$$
S_{i}=\partial\left(\text { core } 3 \text {-disk of } k_{i}^{3}\right), \quad i \leqslant i \leqslant t,
$$


and let $c_{i}$ be the class of $S_{i}$ in $H_{2}(N ; \Lambda)$. The claim will follow from the proof of [11, Lemma 5.7] together with the following observation:

Lemma 7. The classes $c_{1}, \ldots, c_{t}$ generate a subkernel of $K_{2}(N)$.

Proof. Since $S_{1}, \ldots, S_{t}$ are disjointly embedded, we have $\lambda\left(c_{i}, c_{j}\right)=0$ and $\mu\left(c_{i}\right)=$ $0,1 \leqslant i, j \leqslant t$. Also since the inclusion $M^{\prime} \rightarrow U$ induces an isomorphism of fundamental groups, it follows that the inclusion $N-\bigcup_{i} S_{i} \rightarrow N$ also induces an isomorphism of fundamental groups. Consequently, we can find 2-spheres $S_{1}^{\prime}, \ldots, S_{t}^{\prime} \subset$ int $N$ such that $S_{i} \cap S_{j}^{\prime}=\varnothing$ if $i \neq j$ and consists of a single point of transverse intersection if $i=j$. Let $c_{i}^{\prime}$ be the class of $S_{i}^{\prime}$ in $H_{2}(N ; \Lambda)$. It may happen that $c_{i}^{\prime}$ is not contained in $K_{2}(N)$. If this is the case, alter $S_{i}^{\prime}$ as follows: Since $f_{*}^{\prime}: H_{2}\left(M^{\prime} ; \Lambda\right) \rightarrow$ $H_{2}(Y ; \Lambda)$ must be surjective, there exists a class $y_{i}$ in $H_{2}\left(M^{\prime} ; \Lambda\right)$ such that $f_{*}^{\prime}\left(y_{i}\right)=-(F \mid N)_{*}\left(c_{i}^{\prime}\right)$. Represent $y_{i}$ by an immersed 2-sphere $T_{i} \subset$ int $M^{\prime}$. In fact, we can choose $T_{i} \subset$ int $M_{0}^{\prime}$, where $M_{0}^{\prime}=M^{\prime}-\{$ attaching circles of dual 2-handles of $\left.k_{1}^{3}, \ldots, k_{t}^{3}\right\}$. Regard $N$ as obtained from $M_{0}^{\prime}$ by attaching copies of $D^{2} \times S^{2}$. Then $T_{i} \subset N-\bigcup_{i} S_{i}$ and we can assume that $T_{i}$ misses $S_{1}, \ldots, S_{t}$. Add $T_{i}$ to $S_{i}^{\prime}$ via connected sum taken along a suitable arc in $N$ so that $(F \mid N)_{*}\left(c_{i}^{\prime}\right)=0$; i.e., $c_{i}^{\prime}$ is contained in $K_{2}(N)$. Without loss of generality we can assume that the normal bundle of $S_{i}^{\prime}$ in $N$ is trivial. Complete the argument following [11, Chapter 5]. Choose $\mu_{i}$ in $\mu\left(c_{i}^{\prime}\right)$ and make the change of basis

$$
c_{j}^{\prime \prime}=c_{j}^{\prime}-\left(c_{j} \mu_{j}+\sum_{i<j} c_{i} \lambda\left(c_{i}, c_{j}^{\prime}\right)\right) .
$$

We see that $\lambda\left(c_{i}^{\prime \prime}, c_{j}^{\prime \prime}\right)=0, \mu\left(c_{i}^{\prime \prime}\right)=0$ and $\lambda\left(c_{i}, c_{j}^{\prime \prime}\right)=\delta_{i j}, 1 \leqslant i, j \leqslant t$. Thus $c_{1}, \ldots, c_{t}$ generate a subkernel of $K_{2}(N)$.

Conversely, suppose that $(f, b)$ has a solution. Then there exists a normal cobordism $(F, B)$ rel $\partial$ from $(f, b)$ to $\left(f^{\prime}, b^{\prime}\right)$, where $f^{\prime}: P \rightarrow Y$ is a homotopy equivalence. Assume that $F:(V ; M, P) \rightarrow(Y \times I ; Y \times 0, Y \times 1)$. After doing surgery on circles inside int $V$ we may assume without loss of generality that the inclusions $M \rightarrow V, P \rightarrow V$ induce isomorphisms of fundamental groups. Consequently, we can find a handlebody decomposition $V=M \times I \cup k_{1}^{2} \cup \cdots \cup k_{s}^{2} \cup$ $k_{1}^{3} \cup \cdots \cup k_{t}^{3}$ containing handles of index 2 and 3 only. Let $N$ denote the middle-level 4-manifold. Then $N \approx M \# s\left(S^{2} \times S^{2}\right)$. Let

$$
S_{i}=\partial\left(\text { core } 3 \text {-disk of } k_{i}^{3}\right), \quad 1 \leqslant i \leqslant t .
$$

Then the classes of $S_{1}, \ldots, S_{t}$ in $H_{2}(N ; \Lambda)$ generate a subkernel $H_{1} \subset K_{2}(N)$.

Now attach $s$ 2-handles to $W$ along null-homotopic circles in int $M$. Call the resulting 5-manifold $W^{\prime}$. Notice that $\partial W^{\prime}$ can be identified with $N \cup$ \{open collar of $\partial N\}$. Moreover, the image of $H_{3}\left(W^{\prime}, \partial W^{\prime} ; \Lambda\right) \cong H_{3}(W, \partial W ; \Lambda) \oplus \Lambda^{s}$ under the boundary homomorphism $\partial: H_{3}\left(W^{\prime}, \partial W^{\prime} ; \Lambda\right) \rightarrow H_{2}\left(\partial W^{\prime} ; \Lambda\right) \cong H_{2}(N ; \Lambda)$ is the subkernel $H \oplus\left\langle e_{1}, \ldots, e_{s}\right\rangle \subset K_{2}(N)$. Let $H_{2}$ be a subkernel of $K_{2}(N)$ which is dual with respect to intersection pairing.

If it is the case that $\mathrm{H}_{2}$ is representable in $N$, then form $W^{\prime \prime}$ by attaching 3-handles to $W^{\prime}$ along framed 2-spheres in int $N$ (as in $\$ 1$ ) whose classes form a 
basis for $H_{2}$. Then $\partial W^{\prime \prime}=N^{\prime} \cup$ \{open collar of $\left.\partial N\right\}$, where $N^{\prime}$ is obtained from $N$ by surgery. A direct computation reveals that the inclusion $\partial W^{\prime \prime} \rightarrow W^{\prime \prime}$ is a homotopy equivalence. By $\left[9\right.$, Theorem 1.6] $W^{\prime \prime} \approx N^{\prime} \times[0, \infty)$. It follows immediately that the end of $W$ has a collar.

If $\mathrm{H}_{2}$ is stably representable, then after adding sufficiently many cancelling pairs of 2- and 3-handles to the cobordism $V$, we can assume that $\mathrm{H}_{2}$ is in fact representable. In the general case, we do not know whether or not $\mathrm{H}_{2}$ is represent able. Proposition 1 completes the argument.

In order to prove Corollary 5, represent $\beta$ by an element $B$ in $\operatorname{SU}_{r}(\Lambda)$ for some $r$. Perform surgery on the identity map of $M$ to "kill" $r$ null-homotopic circles. We obtain a 2-connected degree-one normal map

$$
(f, b), \text { where } f:\left(M \# r\left(S^{2} \times S^{2}\right), \partial M\right) \rightarrow(M, \partial M) .
$$

The kernel $K_{2}$ is a sum of $r$ standard planes. Regard $B$ as an automorphism of $K_{2}$. If $a_{i}$ denotes the class of $S^{2} \times 1 \subset i$ th-summand $S^{2} \times S^{2}$, then the classes $B\left(a_{1}\right), \ldots, B\left(a_{r}\right)$ generate a subkernel $H$. Construct the manifold $W$ as above using $(f, b)$ and $H$.

Finally, we consider an arbitrary noncompact 5-manifold $W$. For simplicity, we will assume that $\partial W$ is compact (possibly empty). When $\partial W$ is noncompact but diffeomorphic to the interior of a compact manifold, the following arguments can still be used. (See [8].)

Assume that $\varepsilon$ is a tame end of $W$ with vanishing collar obstruction. By [8], we can find neighborhoods $V$ of $\varepsilon$ (in the complement of any prescribed compact set) which satisfy the following conditions:

(i) The neighborhood $V$ is a connected manifold having compact connected boundary and just one end.

(ii) The natural maps $\pi_{1}(\varepsilon) \rightarrow \pi_{1}(V)$ are isomorphisms.

(iii) The inclusion $\partial V \rightarrow V$ induces an isomorphism $\pi_{1}(\partial V) \rightarrow \pi_{1}(V)$.

(iv) The $\Lambda$-module $H_{q}(V, \partial V ; \Lambda)=0$ and $H_{3}(V, \partial V ; \Lambda)$ is a finitely generated free $\Lambda$-module.

In fact, we can choose neighborhoods $V_{1}, V_{2}$ as above with $V_{2} \subset$ int $V_{1}$ such that the cobordism

$$
U=\operatorname{closure}\left(V_{1}-\text { int } V_{2}\right)
$$

has a handlebody-decomposition

$$
U=\partial V_{1} \times I \cup h_{1}^{2} \cup \cdots \cup h_{r}^{2} \cup h_{1}^{3} \cup \cdots \cup h_{s}^{3},
$$

where the classes of the cores of some of the 3 -handles, say $h_{1}^{3}, \ldots, h_{r}^{3}$ generate $H_{3}\left(V_{1}, \partial V_{1} ; \Lambda\right)$. The classes of the remaining cores are mapped isomorphically by

$$
\partial: H_{3}\left(V_{1}, \partial V_{1} ; \Lambda\right) \rightarrow H_{2}\left(\partial V_{1} ; \Lambda\right)
$$

onto image $\partial$. Let $V=V_{2} \cup$ \{dual 2-handles of $\left.h_{r+1}^{3}, \ldots, h_{s}^{2}\right\}$. Denote $\partial V$ by $M$. A straightforward calculation shows that $V$ satisfies conditions (i)-(iv) above and $H_{3}(V, M ; \Lambda)$ is a free $\Lambda$-module of rank $r$ with generators in 1-1 correspondence with $h_{1}^{2}, \ldots, h_{r}^{2}$. Let $a_{1}, \ldots, a_{r}$ be the image of these generators under $\partial: H_{3}(V, M ; \Lambda)$ $\rightarrow H_{2}(M ; \Lambda)$. Then $\lambda\left(a_{i}, a_{j}\right)=0$ and $\mu\left(a_{i}\right)=0,1 \leqslant i, j \leqslant r$. It is not difficult to 
check that the middle-level 4-manifold of $U$ is diffeomorphic to $\partial V_{1} \# r\left(S^{2} \times S^{2}\right)$. (This is also a consequence of [10, Lemma 3.2]). Thus we can find classes $a_{1}^{\prime}, \ldots, a_{r}^{\prime}$ in $H_{2}(M ; \Lambda)$ such that $\lambda\left(a_{i}^{\prime}, a_{j}^{\prime}\right)=0, \lambda\left(a_{i}, a_{j}^{\prime}\right)=\delta_{i j}, \mu\left(a_{i}^{\prime}\right)=0$ for all $i, j$ and the classes $a_{1}, \ldots, a_{r}, a_{1}^{\prime}, \ldots, a_{r}^{\prime}$ generate a free submodule $K$ of $H_{2}(M ; \Lambda)$. In fact, $K$ is a direct summand of $H_{2}(M ; \Lambda)$ which is orthogonal with respect to intersection pairing. By elementary techniques we can construct a Poincaré pair $(Y, X)$ and degree-one normal map $(f, b), f:(M, \partial M) \rightarrow(Y, X)$ such that $f$ is 2-connected and $K_{2}(M)$ is precisely $K$. It is easy to check that all arguments in the proof of Theorem 3 apply to $(f, b)$ and the noncompact 5-manifold $V$.

Addendum. Shortly after I completed this paper, Simon Donaldson [3] proved that there does not exist any smooth closed 1-connected spin 4-manifold with nontrivial definite intersection pairing. This immediately implies the existence of unobstructed surgery problems in dimension four with no solutions. (Such an example can be obtained by attempting to surger the three hyperbolic pairs of the Kummer surface.) Consequently we can now give a handlebody construction, as in $\S 3$ above, of a smooth noncompact 5-manifold $W$ with a single end $\varepsilon$, tame with vanishing collar obstruction, such that $\varepsilon$ has no collar neighborhood in $W$. In fact, $\varepsilon$ is 1 -connected at infinity. Michael Freedman [4], in his recent work on Casson handles, has shown that such an end actually has a topological (i.e., nonsmooth) collar neighborhood.

\section{REFERENCES}

1. S. Cappell and J. Shaneson, On four dimensional surgery and applications, Comment. Math. Helv. 46 (1971), 500-528.

2. F. Connoly, Linking numbers and surgery, Topology 12 (1973), 389-410.

3. S. Donaldson, Self-dual connections and the topology of smooth 4-manifolds, Bull. Amer. Math. Soc. (N.S.) 8 (1983), 81-83.

4. M. Freedman, The topology of four-dimensional manifolds, J. Differential Geom. 17 (1982), 357-453.

5. M. Freedman and F. Quinn, Slightly singular 4-manifolds, Topology 20 (1981), 161-173.

6. J. Milnor, Lectures on the h-cobordism theorem, Princeton Math. Notes, Princeton Univ. Press, Princeton, N. J., 1965.

7. F. Quinn, The stable topology of 4-manifolds, Topology Appl. 15 (1983), 71-77.

8. L. Siebenmann, Doctoral dissertation, Princeton Univ., 1965.

9. On detecting open collars, Trans. Amer. Math. Soc. 142 (1969), 201-227.

10. D. Silver, Finding stable-boundaries for open 5-dimensional manifolds, Amer. J. Math. 105 (1983), 1309-1324.

11. C. T. C. Wall, Surgery on compact manifolds, Academic Press, London and New York, 1970.

12. M. Yamasaki, Whitney's trick for three 2-dimensional homology classes of 4-manifolds, Proc. Amer. Math. Soc. 75 (1969), 365-371.

Department of Mathematics, Dartmouth College, Hanover, New Hampshire 03755

Current address: Department of Mathematics and Statistics, University of South Alabama, Mobile, Alabama 36688 